\title{
Simple multimodality imaging: An easy, rapid, and inexpensive approach to improve non-invasive test accuracy
}

\author{
Todd D. Miller, MD, and Martin Rodriguez-Porcel, $M D^{a}$ \\ a Department of Cardiovascular Diseases, Mayo Clinic, Rochester, MN
}

Received Feb 1, 2016; accepted Feb 1, 2016

doi:10.1007/s12350-016-0445-z

\section{See related article, pp. 711-720}

Cardiac multimodality imaging uses two or more separate imaging technologies to provide information on structure and function. Two imaging modalities that are commonly considered for multimodality imaging include computed tomography (CT) and myocardial perfusion imaging (MPI) by either single-photon emission computed tomography (SPECT) or positron emission tomography (PET). The major goal of multimodality imaging is "to improve the ability to diagnose, guide therapy, or predict outcomes.", Although the concept of multimodality imaging has existed for years, applying this approach in practice has been limited by a number of barriers, including technical limitations related to performing and synthesizing the results of two or more imaging modalities into useful clinical information; longer imaging time; increased radiation exposure; lack of reimbursement; and uncertainty about which patients benefit from this approach. Recent technological advances have led to improvements for both CT and MPI, including shorter imaging times and lower radiation exposure. Industry has capitalized on these advancements and has combined CT with SPECT or PET into single camera systems that can generate a single, co-registered "fusion" or "hybrid" image.

The introduction of camera systems that incorporate multi-slice, "fast" CT cameras have the potential to provide a comprehensive evaluation of the coronary

Reprint requests: Todd D. Miller, MD, Department of Cardiovascular Diseases, Mayo Clinic, Gonda 6-411, 200 First Street, SW, Rochester, MN 55905; miller.todd@mayo.edu

J Nucl Cardiol 2017;24:721-3.

1071-3581/\$34.00

Copyright (C) 2016 American Society of Nuclear Cardiology. arteries by CT angiography. Although appealing, this approach is logistically demanding and expensive, and therefore it is not commonly performed. In practice, the CT component of combination camera systems is most commonly used to perform attenuation correction (AC) of the MPI images. However, the CT images also provide an abundance of information on the anatomy of the chest and upper abdomen, including assessment of coronary artery calcification (CAC).

In this issue of $\mathrm{JNC}$, Patchett et $\mathrm{al}^{2}$ describe the impact of incorporating the assessment of CAC on the diagnostic and prognostic accuracy of stress SPECT MPI. The study population consisted of 1047 patients who underwent a clinically indicated CTAC stress SPECT MPI study over an 18-month period. CAC was assessed subjectively in a simple binary manner as present or absent from the CTAC images. The CAC results were included in the clinical reports. Diagnostic test accuracy was determined in a subset of 109 patients who underwent invasive coronary angiography within 1 year following the SPECT MPI study. Prognostic accuracy was evaluated in the entire study population by medical record review at a median follow-up of 28 months.

CAC was present in almost half ( $47 \%$ ) of the study population and identified higher risk patients (older age, more likely pharmacologic than exercise stress, and insignificant trends for higher prevalence of prior revascularization and risk factors). In the diagnostic cohort, the presence or absence of CAC dramatically influenced the accuracy of SPECT MPI. For patients who were $\mathrm{CAC}(+)$ vs CAC $(-)$, the prevalence of true positive studies was $70 \%$ vs $16 \%(P<.001)$ and false positive studies $15 \%$ vs $68 \%(P<.001)$. These findings had a modest impact on test sensitivity (89\% vs $100 \%)$ and specificity (28\% vs $19 \%$ ) but a major impact on positive predictive value ( $82 \%$ vs $19 \%$ ) and negative predictive value $(42 \%$ vs $100 \%)$. In other words, for patients with positive SPECT MPI, CAD was likely to 
be present only if CAC was positive; conversely, for patients with normal SPECT MPI, CAD could be excluded only if CAC was negative.

CAC by itself was an accurate prognostic variable and also influenced the prognostic accuracy of SPECT MPI. For patients who were CAC (+) vs CAC (-), all individual event rates were higher: mortality $6.0 \%$ vs $0.4 \%(P<.001)$; nonfatal myocardial infarction $5 \%$ vs $0.2 \%(P<.001)$; late revascularization $13 \%$ vs $0.7 \%$ $(P<.001)$. The hazard ratio for the composite outcome endpoint was 22.7 (95\% CI 8.3-62.3) for CAC (+) vs CAC $(-)$. Both CT CAC $(P<.001)$ and SPECT MPI ischemia $(P<.001)$ were independently associated with the outcome endpoint of death or myocardial infarction by multivariable analysis. For survival free of the composite endpoint (74 events), the annual event rate was $<1 \%$ if CAC was absent, regardless of the SPECT MPI results. For patients who were $\mathrm{CAC}(+)$, the annual event rate was approximately $4 \%$ even if SPECT MPI was normal and increased to approximately $9 \%$ if both $\mathrm{CAC}$ and ischemia were present.

These results need to be interpreted within the context of this study's limitations. The most obvious limitation is the small sample size, especially in the coronary angiogram patient cohort $(\mathrm{n}=109)$ and in particular the CAC $(-)$ subset $(\mathrm{n}=25)$. The number of patients in some of the groups used to calculate diagnostic test indices was as few as four patients. Additionally, the derived values for diagnostic test performance were not corrected for post-test verification bias with a large majority ( $85 \%$ ) of the patients in the invasive angiography group having abnormal SPECT MPI. The SPECT images were not interpreted blinded to the CAC results, and therefore the CAC findings may have influenced the interpretation of the SPECT images. In fact, a previous study indicated that knowledge of the CAC score changed the interpretation of SPECT MPI in $37 \%$ of patients. ${ }^{3}$ Given these limitations, the values provided for diagnostic test performance in this study should be interpreted cautiously. Some of the methodology was unconventional, including the interval of 1 year for performance of coronary angiography after SPECT (usually 2-3 months in most studies) and the definition of true-positive SPECT (one coronary territory had to be true-positive) and true-negative SPECT (the entire myocardium had to be true-negative). Furthermore, the approach that was applied to collect follow-up data may have failed to detect all events. Some clinical and imaging results were not provided, including the number of patients who underwent early revascularization and the number of patients who had only fixed SPECT defects. All of these factors could potentially influence the derived values for diagnostic and prognostic test performance, especially in the context of small patient numbers. These issues limit to some degree the robustness of the results of this study and the generalizability of the findings to other study populations.

These limitations, however, do not undermine the major message of this study. Patchett et $\mathrm{al}^{2}$ have demonstrated that the subjective, rapid, and "free" assessment of CAC as a simple dichotomous variable can markedly impact the diagnostic and prognostic accuracy of stress SPECT MPI. As the authors note, the value of multimodality imaging by combining CT CAC and SPECT or PET MPI has been explored by other authors. Most studies have examined the yield of a gatekeeper strategy that first quantifies CAC on a CT scanner followed by stress SPECT MPI performed on a different camera. The goal of this approach is to first perform the lower cost CT CAC test to identify patients who benefit from the higher cost MPI test. These studies have demonstrated an increase in the prevalence of MPI ischemia with higher CAC score. In a recent metaanalysis, Bavishi et $\mathrm{al}^{4}$ reported pooled prevalences of ischemia of $6.6 \%$ for CAC score zero to $23.6 \%$ for CAC score $>400$. These authors noted considerable variation in the prevalence of MPI ischemia reported in different studies, even for low CAC scores but especially for intermediate to high $\mathrm{CAC}$ scores. This wide variation questions whether this approach is advisable for clinical practice.

A small number of studies have examined the impact of CAC on MPI accuracy. Similar to the current study, other authors have reported that CAC improved the diagnostic $c^{5-8}$ and prognostic ${ }^{9-12}$ accuracy of MPI. Part of the appeal of the current study is its simplicity, whereby both images were obtained at nearly the same time on the same camera system. The presence or absence of CAC was determined subjectively and rapidly. Patchett et al's experience with CT-SPECT MPI mirrors our own laboratory's experience with CT-PET MPI. Knowledge of CAC can influence the interpretation (and accuracy) of the MPI findings, especially when the MPI defects are equivocal. Also, there are occasional instances when the clinical and/or stress test findings are highly discrepant from the MPI results, or when severe obstructive CAD is in fact present yet the MPI images are normal or nearly normal. Although other MPI imaging findings such as transient ischemic dilatation ${ }^{13}$ or decrease in rest to stress ejection fraction ${ }^{14}$ can provide clues to the presence of left main and/or 3-vessel CAD, knowledge of CAC may provide another helpful clue to correctly identify these patients. We fully agree with Patchett et al's conclusion that visual assessment of CAC by CT-SPECT or CT-PET imaging cameras, which is essentially free information, should be performed to enhance accuracy. Of course, only nuclear 
cardiology laboratories that have CT MPI camera systems will be able to follow this approach. As imaging technology continues to progress and financial and radiation exposure variables are overcome, hybrid CTMPI imaging that quantifies CAC, provides a non-invasive coronary angiogram, and quantifies myocardial blood flow may one day be the standard for non-invasive assessment of CAD. Until then simple subjective assessment of CAC using CT-SPECT or CT-PET offers a significant step forward for improving imaging accuracy.

\section{References}

1. Weissman NJ, Soman P, Shah DJ. Multimodality imaging: Opportunities and challenges. JACC Cardiovasc Imaging 2013;6:1022-3.

2. Patchett N, Pawar S, Miller E. Visual identification of coronary calcifications on attenuation correction $\mathrm{CT}$ improves diagnostic accuracy of SPECT/CT myocardial perfusion imaging. J Nucl Cardiol 2016. doi:10.1007/s12350-016-0395-5.

3. Mouden M, Ottervanger JP, Timmer JR, Reiffers S, Oostdijk AHJ, Knollema $\mathrm{S}$, et al. The influence of coronary calcium score on the interpretation of myocardial perfusion imaging. J Nucl Cardiol 2013;21:368-74.

4. Bavishi C, Argulian E, Chatterjee S, Rozanski A. Coronary artery calcium score and the frequency of stress-induced myocardial ischemia during myocardial perfusion imaging: A systematic review and meta-analysis. JACC Cardiovasc Imaging 2015 (in press).

5. Schepis T, Gaemperli O, Koepfli P, Namdar M, Valenta I, Scheffel $\mathrm{H}$, et al. Added value of coronary artery calcium score as an adjunct to gated SPECT for the evaluation of coronary artery disease in an intermediate-risk population. $J$ Nucl Med 2007;48:1424-30.

6. von Ziegler F, Brendel M, Uebleis C, Helbig S, Greif M, Ruemmler J, et al. SPECT myocardial perfusion imaging as an adjunct to coronary calcium score for the detection of hemodynamically significant coronary artery stenosis. BMC Cardiovasc Disord 2012;12:1-7.

7. Mouden M, Timmer JR, Reiffers S, Oostdijk AHJ, Knollema S, Ottervanger JP, et al. Coronary artery calcium scoring to exclude flow-limiting coronary artery disease in symptomatic stable patients at low or intermediate risk. Radiology 2013;269:77-83.

8. Brodov Y, Gransar H, Dey D, Shalev A, Germano G, Friedman JD, et al. Combined quantitative assessment of myocardial perfusion and coronary artery calcium score by hybrid 82rb PET/CT improves detection of coronary artery disease. J Nucl Med 2015;56:1345-50.

9. Schenker MP, Dorbala S, Hong ECT, Rybicki FJ, Hachamovitch $\mathrm{R}$, Kwong RY, et al. Interrelation of coronary calcification, myocardial ischemia, and outcomes in patients with intermediate likelihood of coronary artery disease: A combined positron emission tomography/computed tomography study. Circulation 2008;117:1693-700.

10. Chang SM, Nabi F, Xu J, Peterson LE, Achari A, Pratt CM, et al. The coronary artery calcium score and stress myocardial perfusion imaging provide independent and complementary prediction of cardiac risk. J Am Coll Cardiol 2009;54:1872-82.

11. Madsen C, Andersen KF, Zerahan B. High coronary artery calcium score affects clinical outcome despite normal stress myocardial perfusion imaging and normal left ventricular ejection fraction. Coron Artery Dis 2013;24:142-7.

12. Barros MV, Nunes MD, Braga G, Rocha RD, Magalhaes K, Sacaramello F, et al. Role of coronary artery calcium score for risk stratification in patients with non significant perfusion defects by myocardial perfusion SPECT. Cardiol J 2015;22:330-5.

13. Berman D, Kang X, Slomka P, Gerlach J, Yang L, Hayes S, et al. Underestimation of extent of ischemia by gated SPECT myocardial perfusion imaging in patients with left main coronary artery disease. J Nucl Cardiol 2007;14:521-8.

14. Dorbala S, Vangala D, Sampson U, Limaye A, Kwong R, Di Carli MF. Value of vasodilator left ventricular ejection fraction reserve in evaluating the magnitude of myocardium at risk and the extent of angiographic coronary artery disease: A 82rb PET/CT study. J Nucl Med 2007;48:349-58. 